\title{
EL ALPI EN CANTABRIA. ANOTACIONES PRÁCTICAS
}

\author{
LoURdes Royano GuTIÉRREZ \\ (Universidad de Cantabria)
}

\begin{abstract}
RESUMEN
The ALPI was the first complete study of all the peninsular diversities which were studied in linguistics geography. This Atlas was conceived in 1923, under the management of Tomás Navarro. It has a phonetical focussing. It includes 75 maps which analyse from the word «bee» to the word «axle». It was published in 1962.

The volume I, the only one which has appeared, is a phonetical study about the reality observed by the researchers of the age, in spite of not being exhaustive. In this work we present a study of the seventy five ALPI maps and the information proposed, which will be analyse from the variants appreciated in Cantabria's area. The ALPI retakes four types of local speakings in Cantabria: pasiego, montañés, lebaniego y tudanco.
\end{abstract}

Ante la próxima aparición del Atlas Lingüístico y Etnográfico de la provincia de Santander ${ }^{1}$ (ALES), cabe ahora una reflexión sobre otro Atlas Lingüístico español que se ocupaba de Cantabria: el Atlas Lingüístico de la Península Ibérica (ALPI). Este Atlas inacabado, que siguió las directrices del ALF (Atlas lingüístico de Francia) guarda intuiciones significativas sobre el estado de la lengua en Cantabria en la época en que se hicieron los cuestionarios. Sin embargo, la necesidad de renovar y completar los estudios sobre nuestra región ${ }^{2}$,

1 Nótese que se habla de la provincia de Santander, en vez de Cantabria como autonomía, ya que el origen de estos estudios es de 1976.

2 La justificación de estos estudios ha sido ampliamente demostrada por Manuel Alvar en su trabajo «El Atlas lingüístico y etnográfico de la provincia de Santander (España)» Revista de Filologia Española LIX, 1977. Págs. 81-118.

Una nueva edición de este artículo aparece en su libro: Estudios de geografía lingüística. Paraninfo, 1991. Págs. 349-378. 
llevó a un grupo de filólogos, bajo la dirección de Manuel Alvar, a relizar los estudios precisos. Las encuestas de campo del ALES se llevaron a cabo entre 1976 y 1978, en estos días se completa su automatización en el Consejo Superior de Investigaciones Científicas.

En este trabajo presentamos un estudio de los setenta y cinco mapas del ALPI y la información que proponen, que será analizada desde las variantes que se aprecian en la zona de Cantabria ${ }^{3}$.

El ALPI recoge alfabéticamente una lista de palabras, desde «abeja» (mapa $\mathrm{n}^{\circ} 6$ ) hasta «eje» (mapa $\mathrm{n}^{\circ} 75$ ). Para ello, sigue los siguientes pasos:

a) Dado un término, enumera las designaciones en otras lenguas peninsulares.

b) Hace referencia a otros Atlas Lingüísticos y el número de mapa en que se recoge esa palabra.

c) Enumera las designaciones que tiene esa palabra en cada localidad de la provincia. En este tercer punto analizaremos únicamente las designaciones correspondientes a Cantabria.

Ejemplo:

6) ABEJA.

a) Port. ABELHA; cat. ABELLA.

b) ALF 1. ALC 6. AIS 1152. ALL 364.

c) Cantabria:

Este método utilizado en el ALPI ha sido sustituido, en los actuales métodos de cartografía linguiística, por listas onomasiológicas de conceptos; es decir, ya no se parte de una palabra sino de un concepto. Como ejemplo tenemos el propuesto por Alvar':

ALES mapa 8

a) «concepto»: artesa pequeña.

b) «designaciones locales»: cuezo, duerna, -o, masera, amasador, arca, cocio, dezca, desga, artesa, nezca.

Otra característica del ALPI es que en Santander sólo recoge cuatro tipos de hablas locales:

Pasiego (puntos 407, 411)

Montañés (puntos 401, 405)

3 Otro estudioso de la geografía lingüística en Cantabria es Tomás Labrador, investigador del Dpto. de Filología de la Universidad de Cantabria y cuyo proyecto «Lengua y Cultura en Cantabria» le ha llevado a publicar varios artículos (ver bibliografía) y participar en diversos proyectos de investigación, así como a organizar programas culturales para diferentes Universidades Norteamericanas.

4 M. Alvar: «Un ejemplo de Atlas lingüístico automatizado: el ALES» Estudios de geografía lingüística. Paraninfo, 1991. Pág. 380. 


\section{Lebaniego (punto 400)}

Tudanco (punto 406).

El ALPI nació, bajo la dirección de Tomás Navarro Tomás, en 1923. Aunque, en un principio, se empieza a redactar un cuestionario muy variado con dos tipos de preguntas sobre fonética y léxico, debido a problemas de tipo metodológico, se optó por el enfoque fonético, práctica y exclusivamente.

En todos los mapas aparece la Península Ibérica y las Islas Baleares. No se incluyeron Canarias, Ceuta y Melilla, porque, según Navarro Tomás, allí llegó el castellano más tardíamente. Igual que las Islas Azores en Portugal, que se recogerán en el Apéndice $1^{05}$. Tampoco incluye el estudio de los núcleos urbanos. Estaba proyectado el estudio dei habla de alguna capital en el Apéndice $2^{\circ}$. Lo que el ALPI pretende ofrecer es la lengua hablada en pueblos menores y antiguos.

Los equipos de trabajo contaron como principales investigadores con Lorenzo Rodríguez Castellano y Aurelio Espinosa ${ }^{6}$. Empezaron a encuestar en el año 31, en el 36, debido a la guerra civil, se abandonaron los trabajos de campo. Navarro Tomás se lleva los resultados al exilio. Los remitirá en el año 50 al CSIC, porque ve que ya no va a volver. Hay una segunda etapa de encuestas; el primer tomo del ALPI, relativo a Fonética, con 75 mapas lingüísticos que recogen palabras desde la $\mathrm{A}$ hasta la $\mathrm{E}$ en orden alfabético (abeja-eje), aparece en $1962^{7}$.

La selección de informantes seguía los siguientes pasos:

Hombre

ser del lugar

que no tengan un grado de instrucción elevado

(no interferencia norma culta).

Hasta hoy en día, su publicación no ha tenido continuación; es decir, siguen sin publicarse algunos materiales.

La importancia del ALPI viene dada porque, por primera vez, hay mapas de toda la Península con datos fiables, los anteriores son de zonas concretas, como el ALC (Atlas lingüístico de Cataluña) o el ALVA (Atlas lingüístico del Valle de Arán). Por otro lado, el ALPI origina publicaciones de algunos de los encuestadores o del propio director ${ }^{8}$.

En el estudio realizado en Cantabria, podemos observar variedades distintas

5 Estos apéndices están motivados por la distancia que hubo entre la recogida de materiales y su publicación, para actualizar el ALPI.

6 Hay una colaboración con Portugal para la zona portuguesa.

7 Los materiales del ALPI recogidos en los años 52 y 36 fueron publicados al mismo tiempo.

B Navarro Tomás: Capítulos de Geografía Lingüística de la Península Ibérica. Bogotá, Instituto Caro y Cuervo, 1975. 
en el territorio:

Este: influencia del léxico vasco.

Sur: conservador, el castellano más antiguo de lo que se podía pensar.

Sur occidental: no se puede establecer claramente el límite con el asturiano.

Conclusiones a las que hemos llegado:

1. La red de encuesta en el ALPI tiene muchos puntos en Galicia, Asturias, Cantabria, Portugal, Cataluña y Valencia, es decir, frente al centro y Sur donde la densidad es menor 9 .

2. En Cantabria, las formas acabadas en o (cuadro, cuatro, cinco, clavo, cuchillo, cuero, cuévano diario, camino...) se trancriben fonéticamente en -u, como única variante.

3. Retroceso de algunos fenómenos: la aspiración de $-F$ inicial latina. Rodríguez Castellano, en el año $54^{10}$, realizó un estudio sobre los límites de la aspiración. Parece que este fenómeno ha retrocedido, no se puede decir que se conserve la aspirada porque algunas palabras la conserven, son restos fosilizados.

4. El yeísmo avanza directamente. Es una irradiación urbana clarísima.

5. Metafonía: es un fenómeno en retroceso. El refugio último de la metafonía es la Vega de Pas. (y zonas marineras).

6. Se conservan algunos términos prerromanos en léxicos concretos, aunque no pertenecen al acervo de la lengua.

En el ALPI no hay ningún tipo de información etnográfica, que sí se recogerá en el ALES, siguiendo las directrices del AIS (Atlas Italo-Suizo) y lo que se llamó el Metodo «Wörter und Sachen»: palabras y cosas. A partir de este método, se cambian de orientación los Atlas. Se busca la lengua en el entorno cultural que la produce, es decir, por qué las cosas se llaman así, y a qué se llama así. Las palabras se acompañan del dibujo que las representa. Se estudian las relaciones entre denominación y forma.

A partir del AIS, por lo tanto, los atlas serán lingüísticos y etnográficos. En general, perpetúan un cuestionario rural, muy atento a las cosas del campo, a las costumbres. Por ejemplo, muchos nombres se estudian para rastrear creencias, supersticiones, tabúes. Así, por ejemplo, un animal odiado cambia el nombre:

Por ej.: 'mustela: comadreja'. Conservado en Catalán: «mustela» asturiano y aragonés (mustel-ella, mustiliella, mostolilla) ${ }^{11}$.

9 Agradezco a las alumnas Ana Isabel Pacheco Díaz y Rocío San Miguel Montes su colaboración en el recuento fonético realizado en 1991.

to Ver Bibliografía.

11 R. Menéndez Pidal: Manual de Gramática histórica española. Madrid, Espasa Calpe, 1985 , pág. 8 . 
Con la influencia del AIS y del NALF (Nuevo Atlas lingiístico de Francia) nacen los atlas linguísticos españoles nuevos ${ }^{12}$.

ALEA: 1961. Atlas Linguístico y etnográfico de Andalucía.

ALEICan: 1975. Atlas Linguístico y etnográfico de las Islas Canarias.

ALEANR: 1979. Atlas Linguístico y etnográfico de Aragón, Navarra y Rioja.

LMP: 1985. Léxico de los marineros peninsulares. Citado como Atlas ALMP en publicaciones previas.

ALM: Atlas lingüístico del Mediterráneo. Pretendió tener una visión de la forma de hablar de las distintas áreas del Mediterráneo.

ALES: 1976. Atlas Lingüístico y etnográfico de Santander. Actualmente en el Centro de cálculo del CSIC.

ALDC: Atlas Lingüístico del dominio catalán.

Características de estos atlas:

1. Los Atlas linguiísticos españoles son regionales, de pequeño dominio.

2. Estos atlas son etnográficos, hacen referencia a la cultura popular.

3. Además han ampliado su enfoque: se realizarán encuestas en puntos aislados de ciudades. Hacen Dialectología urbana.

4. Utilizaban distintos cuestionarios. El AIS y el NALF aplicaban un cuestionario único, porque la realidad era muy general. Sin embargo, en estos atlas, el cuestionario se limita más en las ciudades omitiendo las preguntas sobre el campo, aperos de labranza, etc... Se adapta el cuestionario a términos urbanos.

\section{ESTUDIO DEL ALPI}

Introducción.

Mapas:

1) Lugares estudiados.

400 Valle de Cabuérniga

401 Yermo

402 Miera

403 Balbacienta

404 Espinama

405 Vega de Liébana

406 Tudanca

407 Vega de Pas

12 Se indica la fecha de comienzo de los Atlas. 
408 Bustantegua

409 Veguilla

410 La Constanza

411 Resconorio

412 Cardaño de Abajo

413 Brañosera

2) Denominación de lugar. Se repiten los nombres con escritura fonética.

3) Denominación de los habitantes.

400 kabwérnigu

402 meráĉu

403 gurje $\theta a ́ n o$

405 lebeniégos

406 tuđánkos

408 pasjégus

4) Areas de las lenguas peninsulares:

Português, Castellano, Català.

(Límites de la diptongación de đ y ŏ)

Nombre dialectal del habla local.

(recogido sólo en algunas localidades)

Cantabria: mõtanés lebenjége, tudánko, pasjégü.

5) Colaboradores de encuesta:

Aurelio M. Espinosa (hijo) $\mathrm{x}$

Lorenzo Rodriguez Castellano $\mathrm{x}$

Anibal Otero

Manuel Sanchís Guarner

Francisco de B. Moll

Luis F. Lindley Cintra.

Armando Nobre de Gusmâo.

Cantabria: $\mathrm{x}$

6) ABEJA. Port. ABELHA; cat. ABELLA.

ALF 1. ALC 6. AIS 1152. ALL 364.

Cantabria: abéha, móska dé mjél, móska dé la mjél, abéxá

7) ABREVADERO. Port. BEBEDOIRO, BEBEDOURO; Cat. ABEURADOR.

ALF 3. ALC 7. AIS (1167) ALL 310. 
Cantabria: bebedéru, pilớn, abrabeđérụ, köínụ

8) ABUELO. Port. AVô; Cat. AVI.

ALF 663. ALC 182. AIS 16. ALR, I, 169.

Cantabria: gwélų, gwílụ, agwélụ, ag wélu.

9) ACERO. Port. AÇO, Cat. ACER.

ALF 8. ALC 14. AIS 401

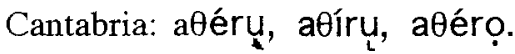

10) AGUA. Port. ÁGUA. Cat. AIGUA

ALF 431, 432. ALC 36. AIS 1037. ALEIC 593.

ALL 631, 632, 633. ALW 30.

11) AGUIJóN (de la abeja). Port. FERRÃO. (Aguilhão) Cat. FIBLÓ (agulló)

ALF 15. AIS 1153. ALL 366.

Cantabria: réspăa, rép̃pa, hịijón, grịihụ, gríxu, harpốn, gíxu.

12) AGÚJA. Port. AGUlHa; cat. AgUlLA.

ALF 14. ALC 29. AIS 1539. ALR,SN,II,523. ALL 658. ALW 1.

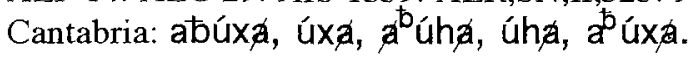

13) AHOGARSE. Port. AFOGAR-SE; Cat. OFEGAR-SE

Cantabria: $a^{g}$ ogaisłe, q̊ogásé, aogá:sé, ahogáse.

14) AIRE. Port. AR; Cat. AIRE

ALEIC 587 a).

Cantabria: áĭri, áiree.

15) AND AR. Port. ANDAR; Cat. CAMINAR (ANAR)

ALC [95]

16) ARAÑ A. Port. ARANHA; Cat. ARANYA

ALF 50. ALC 137. AIS 485. ALL 577. ALG 69.

Cantabria: arạnón, aránga.

17) ÁRBOLES. Port. ÁRVORES; Cat. ARBRES

ALF 52. ALC 141. AIS 1251,[533]. ALL [428]. ALG [88]

18) ASA. Port. ASA; Cat. ANSA. 
19) AVISPA. Port. VESPA; Cat. VESPA

ALF 672. AIS 463. ALL 502. ALW 48. ALG 49.

Cantabria: abrjéspạ, agrjéṡpa, bjésporă, grjéspa, abjépra, abríspa

20) AYER. Port. ONTEM; Cat. AHIR

ALF 695. ALC 33. AIS 349. ALL 1309.

21) AYUNAR. Port. JEJUAR; Cat. DEJUNAR.

ALF [721]. ALC 611. AIS 802.

22) AZADA. Port. ENXADA; Cat. AIXADA.

ALF 1763. ALC 43. AIS 1428. ALR,SN,I 28. ALL 158.

Cantabria: aӨá, aӨádá.

23) BAILE. Port. BAILE; Cat. BALL.

ALF [377]. ALC 299. AIS 755.

24) BAÚL. Port. BAÚ; Cat. BAGUL.

ALF 1450. ALC 196. ALR II,I, pág. 136

Cantabria: baụ́l, húçø, árkạ, mứndu, bagúl.

25) BLANCO. Port. BRANCO; Cat. BLANC.

ALF 135. ALC 273. AIS 1575.

Cantabria: blánku.

26) BOCA. Port. BOCA; Cat. BOCA.

ALF 151. ALC 281. AIS 104. ALL 1079.

27) BRAZO. Port. BRAÇO; Cat. BRAÇ.

ALF 171. ALC [307]. AIS 144. ALR,II,48

Cantabria: brá $\theta$ ü.

28) BUEY. Port. BOI; Cat. BOU.

ALF 141. ALC, 305. AIS 1042. ALL 276 add. ALW 4.

Cantabria: gwể.

29) CABAllo. Port. CAVAlO; Cat. CAVAlL.

ALF 269. ALC 449. AIS 1062. ALR,SN,II,294. ALL 311. 
Cantabria: kabáyü, kabálüu, kaválúlu.

30) CABEZA. Port. CABEÇA; Cat. CAP.

ALF 1300. ALC 399. ALR,I,5. ALL 1053. ALW 95.

3I) CAER. Port. CAIR; Cat. CAURE.

ALF 1311. ALC 448.

Cantabria: kayềp, kaếf.

32) CAJA. Port. CAIXA; Cat. CAIXA.

ALF 197. ALC 359. AIS 903.

Cantabria: káhă, káxạ.

33) CAMINO. Port. CAMINHO; Cat. CAMÍ.

ALF 262. ALC 378. ALL 837.

Cantabria: kamînụ.

34) CÁNTARo. Port. CâNTARO; Cat. CÀNTER,CÀNTIR.

ALF 1526. ALC 395.

Cantabria: kángterụ, kántặra, kángtera, kántạro.

35) CAÑA. Port. CANA; Cat. CANYA.

ALF 1166. ALC 397. AIS 524 add. ALR,II,230. ALG 186.

Cantabria: káña, kanăbéră.

36) CARACOL. Port. CARACOL; Cat. CARGOL,CARAGOL.

ALF 481. ALC 418. AIS 459. ALEIC 590. ALL 579. ALG 67.

Cantabria: karạkộl, karikót.

37) CASTILlo. Port. CASTELO; Cat. CASTELL.

ALF 252. ALC 445. AIS 817 add.

Cantabria: kastílü, kaștíyu.

38) CAUSA. Port. CAUSA; Cat. CAUSA.

39) CAZADOR. Port. CAÇADOR; Cat. CAÇADOR.

ALF 249. AIS 518. ALW 14.

40) CAZUEla. Port.CAÇOILA; Cat. CASSOLA

ALF 202. ALC 441 
41) CEJAS. Port. SOBRANCELHAS; Cat. CELLES.

ALF 1257. ALC 456. AIS 102. ALR ,I,21. ALEIC 76. ALL 1057.

42) CEPA. Port. CEPA; Cat. CEP.

ALF 1780. ALC 461. AIS 1305. ALL 193.

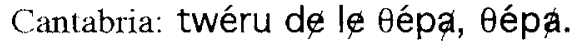

43) CEREzA. Port. CEREJA; Cat. CIRERA.

ALF 217. ALC 476. AIS 1282. ALEIC 285. ALL 466 add. ALW 8. ALG [98].

44) CERROJO. Port. FERROLHO; Cat. FORRELLAT.

ALF 1374. ALC 850. AIS, 888. ALR,II 251. ALL 699.

Cantabria: Өeróhu, berớhu, beróxu, beróxoxo.

45) CHINCHE. Port. PERCEVEJO; Cat. XINXA.

ALF 1105. AIS 473. ALL 533. ALG 59.

46) CINCHA. Port. CILHA; Cat. CINGLA.

ALC 472.

47) CINCO. Port. CINCO; Cat. CINC.

ALF 289. ALC 470. AIS 286. ALW 19.

Cantabria: $\theta i ̣$ ịụ.

48) CLAVO. Port. (CRAVO=»clavel»); Cat. CLAU.

ALF 340. ALC 488. AIS 230. ALR,II,I pág.126. ALL 246.

ALW 21.

Cantabria: klábu, klá ú.

49) COCINA. Port. COZINHA; Cat. CUINA.

ALF 366. ALC 595. AIS 942. ALL 703.

50) COJO. Port. COXO; Cat. COIX.

ALC 505. AIS 191. ALR,I,71. ALL 1102.

Cantabria: kóhư, kóxụ.

51) COZ. Port. COICE,COUCE; Cat. COÇ.A.

52) CRESTA. Port. CRISTA; Cat. CRESTA. ALF 351. ALC 578. AIS 1127. ALL 334 add. 
53) CRIN. Port. CRINA; Cat. CRINA.

ALF 357. AIS 1064.

Cantabria: klína, eqklín, klịn.

54) CRUZ. Port. CRUZ; Cat. CREU.

ALF 363. ALC 579. AIS 790.

55) CUADRADO. Port. QUADRADO; Cat. QUADRAT.

Cantabria: kwađráŭ.

56) CUATRO. Port. QUATRO; Cat. QUATRE.

ALF 1112. AIS 285.

Cantabria: kwátrụ.

57) CUCHARA. Port. COlHER; Cat. CULLERA.

ALF 367. ALC 600. AIS 982. ALR [S.N. II,419]

Cantabria: kuĉár, kuĉáf, kộcár.

58) CUCHILlo. Port. FACA (CUTELO);

Cat. GANIVET (COLTELL).

ALF 341. AIS 979. ALL 634.

Cantabria: koĉilu, koĉíyu, kuĉiú, koĉilụ.

59) CUERO. Port. COIRO,COURO; Cat. CUIR,CUIRO.

ALF 368. AIS 207. ALL 1138. ALW 26.

Cantabria: kwérụ, kwírụ.

60) CUÉVANO.Port. CESTO(COVO); Cat.COVE.

ALF 307. ALC 574. ALL 24.

Cantabria: kwể anụ, kwénư, kwébano.

61) CUÑADOS.Port. CUNHADOS; Cat.CUNYATS.

ALC[602]. AIS 28. ALR[I,263].

Cantabria: kwnáus, kunáuns.

62) (no quiere) DECIRLO. Port.(nao quer) DIZE-LO.

Cat.(el parenostre, no vol) DIR-LO.

ALF [1526]. ALC [641]

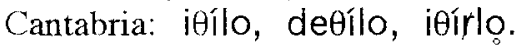


63) DEDO.Port. DEDO; Cat. DIT.

ALF 416. ALC 651. AIS 153. ALR ,I,50. ALL 1095.

Cantabria: déu, déđu, déđo.

64) DERECHA. Port. DIREITA; Cat. DRETA.

ALF 472. AIS 148. ALR II,I,pág.26. ALL 1093.

65) DESBOCADO. Port. DESENFREADO (DESBOCADO); Cat. DESBOCAT.

Cantabria: eżbokáun, de bokáu, exbokáun.

66) DESNUDO. Port. NU; Cat.NU.

AIS 670.

Cantabria: desnúđư, de $e^{z}$ ú, de núgu, aznúgu, eznúđu, dernúđu.

67) DEUDAS. Port. DÍVIDAS; Cat. DEUTES.

ALF 1534. AIS 283.

68) DIARIO. Port. DIÁRIO; Cat. DIARI.

ALF 730. AIS 769.

Cantabria: djárjụ

69) DIENTE. Port. DENTE; Cat. DENT.

ALC 617. AIS [107]. ALR,I,29. ALL [1082 add.]. ALW 27.

70) DIEZ. Port. DEZ; Cat. DEU.

ALF 412. AIS 288.

71) DOCE. Port. DOZE; Cat. DOTZE.

ALF 424. ALC 670. AIS 290.

72) LOS DOMINGOS. Port. OS DOMINGOS; Cat. ELS DIUMENGES.

ALF [405]. ALC [653]. AIS [335]. ALL [880]. ALW [29].

73) ¿DÓNDE?. Port. ONDE?; Cat. ON?.

ALF 25. ALL [1317].

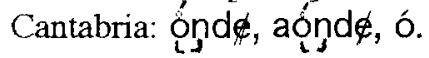

74) DULCE. Port. DOCE; Cat. DOLÇ.

ALF 421. ALR II,I pág.14. 
75) EJE. Port. EIXO; Cat. EIX.

ALF 484.

Cantabria: é̀ $x e$, ếne.

\section{Refer encias bibliográficas:}

ALCALDE DEL RIO, H.: Contribución al léxico montañés. Santander, 1933.

ALVAR, Manuel: Los nuevos atlas lingüísticos de la Romania. Granada, 1960.

- : «Los atlas linguiísticos de España» Presente y futuro de la lengua española.Tomo 1. Instituto de Cooperación Iberoamericana. Madrid, 1963. Págs. 417-426.

- : «El Atlas Linguístico y Etnográfico de la provincia de Santander» RFE LIX, 1979. Págs. 81-118.

- : Estudios de geografía lingüística. Madrid, Paraninfo, 1991.

BARTOLOME SUAREZ, Antonio: Aforismos, giros y decires en el habla montañesa. Servicio de Publicaciones de la Universidad de Cantabria, 1993

CALDERON ESCALADA, J.: «Voces, en su mayor parte de cosas, de uso corriente en estos valles altos de la provincia de Santander, que no están recogidas en el Diccionario de la Lengua española». Boletín de la Real Academia española, XXV, 1946 Págs. 379-397.

- : «Voces, en su mayor parte de cosas, de uso corriente en los valles altos de la provincia de Santander, que no están recogidas en el Diccionario de la Lengua española». Boletín de la Real Academia española, XXXIII, 1953. Págs. 295-304.

CAMINO Y AGIRRE, G.: «Hilanderas y telares en la Montaña». La revista de Santander, 1932-33.

COSERIU, Eugenio: «La geografía linguística» El hombre y su lenguaje. Madrid, Gredos, Biblioteca Románica Hispánica, estudios y ensayos 272. 1977. Págs. 103-158.

COSSIO, José $\mathrm{M}^{\mathrm{a}}$ de: «Aportación al léxico montañés». Boletín de la Biblioteca Mnéndez Pelayo IX, 1927. Págs. 115-122. (Vocabulario de Tudanca)

DAUZAT, A.: La géographie linguistique. Paris, Flammarion, 1922.

ENRIQUEZ, E.: «Análisis automático de la información fónica contenida en los Atlas linguísticos». Lingüística española actual VII, 1. Madrid, Instituo de Cooperación Iberoamericana, 1986. Págs. 93-131.

GARCIA LOMAS, Adriano: Estudio del dialecto popular montañés. Fonética, etimologías y glosario de voces. San Sebastián, 1922.

- : El lenguaje popular de las montañas de Santander. Fonética, recopilación de voces, refranes y modismos. Santander, 1949.

GONZALEZ CAMPUZANO, Juan: «Apuntes para un vocabulario montañés». Revisadas y anotadas por E. de Huidobro. Boletín de la Biblioteca Menéndez. 
Pelayo II, 1920. Págs. 3-10 y 59-68.

GONZALEZ ECHEGARAY, J. / DIAZ GOMEZ, A.: Manual de etnografía cántabra. Santander, Estudio, 1988.

HUIDOBRO, Eduardo de: Palabras, giros y bellezas del lenguaje popular de la Montaña. Santander, 1907.

LABRADOR GUTIERREZ, Tomás: «Variantes léxicas en la toponimia menor de la Comarca de la Liébana» XVII Simposio de S.E.L. Murcia, 1987.

- : «En torno al género de los nombres». Letras de Deusto. Vol. 18, $\mathrm{n}^{\circ} 42$, 1988. Págs. 21-54

— : «Hipótesis sobre toponimia menor en Tresviso» XIX Simposio de S.E.L. Salamanca, 1989.

-: «MOLINO, MOLINA y sus derivados y compuestos en la microtoponimia española». Proceedings of theXVII th International Congress of Onomastic Sciences, Vol 2. The University of Helsinki, Finlandia, 1990. Págs. 71-79.

- : «Constantes, unidad y variedad en toponimia» $X$ Congreso Internacional de ALFAL, Veracruz, Méjico, 1993. (en prensa)

MONTES, J.J.: «Dialectología y sociolinguiística: algunas ideas sobre sus interrrelaciones» Lingüística española actual VIII, 1. Madrid, Instituto de Cooperación Iberoamericana, 1986. Págs. 133-141.

NAVARRO TOMAS, Tomás: ALPI. Atlas lingüístico de la Península Ibérica.I

Fonética, Consejo Superior de Investigaciones Científicas. Madrid, 1962.

- : Capítulos de geografia lingüística de la Península Ibérica. Bogotá, Instituto Caro y Cuervo, 1975.

PENNY, Ralf J.: El habla pasiega: ensayo de dialectologia montañesa. Londres, 1969.

--: «Esbozo de un Atlas de Santander». Lingüística española actual VI, 1984. Págs. 123-181.

RODRIGUEZ CASTELLANO, Lorenzo: «Estado actual de la «h» aspirada en la provincia de Santander» Archivum IV, 1954. Págs. 435-437.

- : «Algunas precisiones sobre la metafonía de Santander y Asturias» Archivum IX, 1959. Págs. 236-248.

ROYANO GUTIERREZ, Lourdes: «Algunas variaciones léxicas en un relato de Miguel Angel Asturias» Congreso XX Aniversario de la Sociedad Española de Lingüística. Madrid, Gredos.1990. Págs. 1079-1087.

- : «Nuevo enfoque del acto comunicativo. Variaciones linguísticas en un relato de Miguel Angel Asturias». El español de América. Actas del III Congreso Internacional. Universidad de Valladolid- Junta de Castilla y León. 1991. Págs. 1639-1649.

SALVADOR, G.: Estudios dialectológicos. Madrid, Paraninfo, 1987.

VVAA: Museos para aprender. Aula de etnografía de la Universidad de Cantabria, 1992. 